\title{
Detecting Critical Configuration of Six Points
}

\author{
Yihong WU and Zhanyi HU \\ National Laboratory of Pattern Recognition, Institute of Automation, \\ Chinese Academy of Sciences, P.O. Box 2728, Beijing 100080, P.R. China \\ $\{$ yhwu, huzy\}@nlpr.ia.ac.cn \\ http://www.nlpr.ia.ac.cn/English/rv
}

\begin{abstract}
When space points and camera optical center lie on a twisted cubic, no matter how many corresponding pairs there are from space points to their image points, camera projection matrix cannot be uniquely determined, in other words, the configuration of camera and space points in this case is critical for camera parameter estimation. In practice, it is important to detect this critical configuration before the estimated camera parameters are used. In this work, a new method is introduced to detect this critical configuration, which is based on an effective criterion function constructed from an invariant relationship between six space points and their corresponding image points. The advantage of this method is that no explicit computation on camera projection matrix or optical center is needed. Simulations show it is quite robust and stable against noise. Experiments on real data show the criterion function can be faithfully trusted for camera parameter estimation.
\end{abstract}

\section{Introduction}

Projective geometric invariant plays an important role in computer vision. Since 1994, there have been many studies on the invariant relationship between six space points and their image points [2-4, 7, 9-13]. The invariant relationship can be applied to 3D reconstruction, object recognition, robot vision and so on as shown in the literature.

On the other hand, estimation of camera parameters is a key problem in 3D reconstruction. One of the popular methods for this problem is to recover the camera parameters from at least six pairs of image points and their corresponding spatial points with known coordinates [1]. By using this method, many degenerate configurations may occur. There are systematic analyses for these degenerate configurations in Chapter 21 of [6], which consist of two cases: incidence case and non-incidence case. The incidence case is that some of the space points are collinear or coplanar, or some of the space points and the camera optical center are collinear or coplanar. The non-incidence case is that the space points and the camera optical center lie on a proper twisted cubic, of which no three space points are collinear and no four space points are coplanar, also no two space points are collinear and no three space points are coplanar with the camera optical center. How to detect these degenerate configurations? This 
is the problem considered here. For the incidence case, it is easy to detect by determining the linearly dependent relations among the space points or the image points. However, for the non-incidence case, namely the case that the space points and the camera optical center lie on a proper twisted cubic, it is difficult to detect. The method of [16] can detect this degenerate configuration. But, estimation of the camera optical center is needed at first, and also it is sensitive to noise.

Detecting degenerate configurations is important because if a spatial configuration is degenerate mathematically but the noise from the measured image makes it non-degenerate, any estimation under such configuration is useless [15]. For camera parameter estimation, the data from those degenerate configurations just mentioned are critical and can result in dangerous recovered camera parameters.

Our method in this paper can effectively detect the degenerate configuration in the non-incidence case. This degenerate configuration in the non-incidence case (camera and space points lie on a proper twisted cubic) is called twisted cubic degenerate configuration, or twisted cubic configuration in the following.

By using brackets like in [2-5], we establish the invariant relationship between six space points and their images under a perspective view for the twisted cubic configuration. This configuration is different from the previous general one $[2-4$, 7, 9-13]. The established invariant relationship is free of the camera optical center and camera projective matrix. From it, then an algorithm based on a weighed criterion function is proposed to detect the twisted cubic degenerate configuration. Simulations and experiments on real data are performed, which show the proposed algorithm is quite stable against noise and the criterion function is reasonably useful in practice.

The organization of the paper is as follows. Some preliminaries are listed in Section 2. Section 3 reports the invariant relationship between six space points and their images under a perspective view for the twisted cubic configuration, and then elaborates the algorithm to detect the twisted cubic degenerate configuration for camera parameter estimation. Experiments are shown in Section 4 , and Section 5 are some conclusions.

\section{Preliminaries}

In this paper, a bold capital letter denotes either a homogeneous 4-vector or a matrix, a bold small letter denotes a homogeneous 3-vector, a bracket "[ ]" denotes the determinant of vectors in it. And in addition, we assume that no three image points are collinear, no four space points are coplanar (so the brackets on the image and space points are always nonzero).

Under the pinhole camera, a space point $\mathbf{M}_{i}$ is projected to a point $\mathbf{m}_{i}$ in the image plane by:

$$
s_{i} \mathbf{m}_{i}=\mathbf{K}(\mathbf{R}, \mathbf{t}) \mathbf{M}_{i}, \quad i=1 . .6,
$$

where $\mathbf{K}$ is the $3 \times 3$ matrix of camera intrinsic parameters, and $\mathbf{R}, \mathbf{t}$ are a $3 \times 3$ rotation matrix and a $3 \times 1$ translation vector, $s_{i}$ is a nonzero scalar. If $s_{i}$ 
were zero, then $\mathbf{M}_{i}$ could not be projected to the image plane. We assume that camera optical center $\mathbf{O}$ and six space points $\mathbf{M}_{i}$ are not at infinity throughout this paper.

Then under (1), the established relation in [4] between bracket on image points and bracket on space points is:

$$
s_{i} s_{j} s_{k}\left[\mathbf{m}_{i}, \mathbf{m}_{j}, \mathbf{m}_{k}\right]=\operatorname{det}(\mathbf{K})\left[\mathbf{M}_{\mathbf{i}}, \mathbf{M}_{\mathbf{j}}, \mathbf{M}_{\mathbf{k}}, \mathbf{O}\right] .
$$

We will use (2) later.

In the following, for the notational convenience, if no ambiguity can be aroused, $\mathbf{M}_{i}, i=1 . .6$ will be simply denoted as $\mathbf{1}, \mathbf{2}, \mathbf{3}, \mathbf{4}, \mathbf{5}, \mathbf{6}$, and the commas in the brackets will be omitted.

There is a unique proper twisted cubic passing through six space points $\mathbf{1}, \mathbf{2}, \mathbf{3}, \mathbf{4}, \mathbf{5}, \mathbf{6}$ with no three collinear and no four coplanar. Any point $\mathbf{X}$ is on this twisted cubic if and only if [16]:

$$
\left\{\begin{array}{l}
\frac{[\mathbf{1 2 4 6}][\mathbf{1 3 5 6}]}{[\mathbf{1 2 3 6}][\mathbf{1 4 5 6}]}=\frac{[\mathbf{1 2 4 X}][\mathbf{1 3 5 X}]}{[\mathbf{1 2 3 X}][\mathbf{1 4 5 X}]}, \\
\frac{[\mathbf{1 2 4 6}][\mathbf{2 3 5 6}]}{[\mathbf{1 2 3 6}][\mathbf{2 4 5 6}]}=\frac{[\mathbf{1 2 4 X}][\mathbf{2 3 5 X}]}{[\mathbf{1 2 3 X}][\mathbf{2 4 5 X}]}, \\
\mathbf{X} \text { is not on the line through } \mathbf{1 , 2} .
\end{array}\right.
$$

The above representation is not unique as a result that the one after a permutation of $\mathbf{1}, \mathbf{2}, \mathbf{3}, \mathbf{4}, \mathbf{5}, \mathbf{6}$ is also a representation of the same twisted cubic.

We can see that each bracket in the first equation of (3) has the point $\mathbf{1}$. The geometric meaning of this equation is that $\mathbf{1}, \mathbf{2}, \mathbf{3}, \mathbf{4}, \mathbf{5}, \mathbf{6}, \mathrm{X}$ lie on a quadric cone with $\mathbf{1}$ as the vertex [16]. Similarly, the second equation of (3) means that $\mathbf{1}, \mathbf{2}, \mathbf{3}, \mathbf{4}, \mathbf{5}, \mathbf{6}, \mathrm{X}$ lie on a quadric cone with $\mathbf{2}$ as the vertex. This is consistent with the theorem in [14] that a twisted cubic can be the intersection of two quadrics.

\section{Recognizing Critical Configuration of Six Points}

\subsection{Invariant Relationship for the Twisted Cubic Configuration}

Proposition 1. The camera optical center $\mathbf{O}$ lies on the proper twisted cubic passing through $\mathbf{1}, \mathbf{2}, \mathbf{2}, \mathbf{4}, \mathbf{5}, \mathbf{6}$ if and only if

$$
\left\{\begin{array}{l}
{\left[\mathbf{m}_{1} \mathbf{m}_{2} \mathbf{m}_{3}\right]\left[\mathbf{m}_{1} \mathbf{m}_{4} \mathbf{m}_{5}\right][\mathbf{1 2 4 6}][\mathbf{1 3 5 6}]} \\
-\left[\mathbf{m}_{1} \mathbf{m}_{2} \mathbf{m}_{4}\right]\left[\mathbf{m}_{1} \mathbf{m}_{3} \mathbf{m}_{5}\right][\mathbf{1 2 3 6}][\mathbf{1 4 5 6}]=0, \\
{\left[\mathbf{m}_{1} \mathbf{m}_{2} \mathbf{m}_{3}\right]\left[\mathbf{m}_{2} \mathbf{m}_{4} \mathbf{m}_{5}\right][\mathbf{1 2 4 6}][\mathbf{2 3 5 6}]} \\
-\left[\mathbf{m}_{1} \mathbf{m}_{2} \mathbf{m}_{4}\right]\left[\mathbf{m}_{2} \mathbf{m}_{3} \mathbf{m}_{5}\right][\mathbf{1 2 3 6}][\mathbf{2 4 5 6}]=0 .
\end{array}\right.
$$

After a permutation of $\mathbf{1}, \mathbf{2}, \mathbf{3}, \mathbf{4}, \mathbf{5}, \mathbf{6}$ and the corresponding image points, this equation system is still the invariant relationship of $\mathbf{1 , 2}, \mathbf{3}, \mathbf{4}, \mathbf{5}, \mathbf{6}, \mathbf{O}$ lying on the same twisted cubic, but is not independent of the above one. 
Proof. Proposition 1 can be obtained by (3) and (2) as follows. If $\mathbf{O}$ lies on the twisted cubic through $\mathbf{1}, \mathbf{2}, \mathbf{3}, \mathbf{4}, \mathbf{5}, \mathbf{6}$, then $\mathbf{O}$ satisfies (3), further by (2), we have:

$$
\left\{\begin{array}{l}
\frac{[\mathbf{1 2 4 6}][\mathbf{1 3 5 6}]}{[\mathbf{1 2 3 6}][\mathbf{1 4 5 6}]}=\frac{[\mathbf{1 2 4 O}][\mathbf{1 3 5 O}]}{[\mathbf{1 2 3 O}][\mathbf{1 4 5 O}]}=\frac{\left[\mathbf{m}_{1} \mathbf{m}_{2} \mathbf{m}_{4}\right]\left[\mathbf{m}_{1} \mathbf{m}_{3} \mathbf{m}_{5}\right]}{\left[\mathbf{m}_{1} \mathbf{m}_{2} \mathbf{m}_{3}\right]\left[\mathbf{m}_{1} \mathbf{m}_{4} \mathbf{m}_{5}\right]} \\
\frac{[\mathbf{1 2 4 6}][\mathbf{2 3 5 6}]}{[\mathbf{1 2 3 6}][\mathbf{2 4 5 6}]}=\frac{[\mathbf{1 2 4 O}][\mathbf{2 3 5 O}]}{[\mathbf{1 2 3 O}][\mathbf{2 4 5 O}]}=\frac{\left[\mathbf{m}_{1} \mathbf{m}_{2} \mathbf{m}_{4}\right]\left[\mathbf{m}_{2} \mathbf{m}_{3} \mathbf{m}_{5}\right]}{\left[\mathbf{m}_{1} \mathbf{m}_{2} \mathbf{m}_{3}\right]\left[\mathbf{m}_{2} \mathbf{m}_{4} \mathbf{m}_{5}\right]}
\end{array}\right.
$$

Notice that in (3), there is another condition such as: $\mathbf{X}$ is not on the line through $\mathbf{1}$ and $\mathbf{2}$. Here for the optical center $\mathbf{O}$, this additional condition is unnecessary because if $\mathbf{O}$ is on the line through $\mathbf{1}$ and $\mathbf{2}$, then $\mathbf{m}_{1}$ and $\mathbf{m}_{2}$ become one point, which is contrary to our assumption that no three image points are collinear.

Because the representation (3) is independent of the order of $\mathbf{1}, \mathbf{2}, \mathbf{3}, \mathbf{4}, \mathbf{5}, \mathbf{6}$, the invariant relationship (4) is also independent of the order of them and their corresponding image points. Proposition 1 is proved.

Each ratio in (5) is a cross ratio [5]. Therefore, (4) is an invariant relationship between space points and image points equivalent to (5).

By the last paragraph of Section 2 or [14], we know the image points $\mathbf{m}_{i}, i=$ 1..6, from the twisted cubic configuration are con-conic. But, this is not a sufficient condition for this configuration [14].

We have established the above invariant relationship between six space points and their images under a perspective view when the optical center and the space points lie on a twisted cubic. The number of the equations describing the relationship is two, which is different from the number one for the previous general configuration $[2-4,7,9-13]$. The reason is just from the degeneracy of the twisted cubic configuration [17].

This invariant relationship can be easily extended to invariant relationship between two perspective views that the two camera optical centers and six space

points lie on a twisted cubic. Then the result can be used to detect critical data for computing fundamental matrix or epipoles $[6,8]$.

\subsection{Establishing an Algorithm to Detect Twisted Cubic Degenerate Configuration of Six Points}

Detecting the twisted cubic degenerate configuration is important because the data for camera parameter estimation from the degenerate configuration is critical and can result in useless recovered camera parameters.

We apply the established invariant relationship to detect the twisted cubic degenerate configuration of six points. The method is based on a criterion function that can be faithfully trusted for camera parameter estimation from six points in practice.

Notice that in the invariant relationship (4) of the twisted cubic case, $\mathbf{m}_{6}$ does not occur. And, by the last paragraph of Section 2, we know that the 
first equation of (4) is the cone with $\mathbf{1}$ as the vertex, the second equation of (4) is the cone with $\mathbf{2}$ as the vertex. Thus, the two equations are denoted as $g_{1,(24,35)}=0, g_{2,(14,35)}=0$, which can be criterion functions to recognize the twisted cubic degenerate configuration. But, stability to noise is much affected by the order of space points and image points. So, we are to consider more equations after changing the orders of space points and their corresponding images. We do a permutation on $\mathbf{1}, \mathbf{2}, \mathbf{3}, \mathbf{4}, \mathbf{5}, \mathbf{6}$ and their corresponding images in $g_{1,(24,35)}$

or $g_{2,(14,35)}$ and denote the corresponding result as $g_{i,(j k, p q)}$. For each of such permuted functions, we also assign a weigh to it, and then the criterion function on $\mathbf{1}, \mathbf{2}, \mathbf{3}, \mathbf{4}, \mathbf{5}, \mathbf{6}$ and their image points is constructed as:

$$
f=\frac{1}{90} \sum_{i=1}^{6} \sum_{\sigma \in S} \frac{1}{W_{i, \sigma}^{2}} g_{i, \sigma}^{2},
$$

where $S=\{(j k, p q),(j p, k q),(j q, k p),(j k, p l),(j p, k l),(j l, k p),(j k, q l),(j q, k l)$, $(j l, k q),(j p, q l),(j q, p l),(j l, p q),(k p, q l),(k q, p l),(k l, p q)\}$ has 15 elements and $\{i, j, k, p, q, l\}=\{1,2,3,4,5,6\}$. According to our experience from extensive experiments, the weigh $W_{i, \sigma}$ is taken as the mean of the absolute values of the two terms in $g_{i, \sigma}$ in this work.

Now, we can propose a two-step algorithm to determine whether six space points and the camera optical center lie on a proper twisted cubic or not, where no four of the space points are coplanar, and no three of the image points are collinear.

Step 1. Compute the value of the criterion function $f$ on the six space points $\mathbf{1}, \mathbf{2}, \mathbf{3}, \mathbf{4}$, $\mathbf{5 , 6}$ and their corresponding image points.

Step 2. Let $\epsilon$ be a preset threshold, and determine whether $f<\epsilon$. If yes, then $\mathbf{1 , 2}, \mathbf{3}, \mathbf{4}, \mathbf{5}, \mathbf{6}$ and the camera optical center lie on a proper twisted cubic. Otherwise, they are not on a twisted cubic.

It is clear that the criterion function $f$ is only on the image and space points, and in it there is no any computation on the camera optical center or projective matrix. $f$ can let us efficiently know whether space points and camera lie on the same twisted cubic or not, also it can be faithfully trusted for camera parameter estimation from six points in practice as shown in real experiments. According to our experience from extensive experiments, the threshold $\epsilon$ is taken as $1.1 \mathrm{in}$ this work.

\section{Experiments}

\subsection{Simulations}

We perform experiments on simulated data to test the stability of the proposed algorithm in the following. The world coordinate system is taken as the camera coordinate system. The simulated camera intrinsic parameters are:

$$
\mathbf{K}=\left(\begin{array}{ccc}
1000 & 0 & 512 \\
0 & 900 & 384 \\
0 & 0 & 1
\end{array}\right)
$$


then we generate the images of seven space points $\mathbf{1}, \mathbf{2}, \mathbf{3}, \mathbf{4}, \mathbf{5}, \mathbf{6}, \mathbf{7}$ such that $\mathbf{1}, \mathbf{2}, \mathbf{3}, \mathbf{4}, \mathbf{5}, \mathbf{6}, \mathrm{O}$ do not lie on a twisted cubic, and $\mathbf{1}, \mathbf{2}, \mathbf{3}, \mathbf{4}, \mathbf{5}, \mathbf{7}, \mathrm{O}$ do lie on a twisted cubic. The Gaussian noise with mean 0 and standard deviation ranging from 0 to 6 pixels is directly added to each image points, and then $I_{1}$ : the value of the criterion function of $f$ on $\mathbf{1}, \mathbf{2}, \mathbf{3}, \mathbf{4}, \mathbf{5}, \mathbf{6}$ and their image points and $I_{2}$ : the value of the criterion function of $f$ on $\mathbf{1}, \mathbf{2}, \mathbf{3}, \mathbf{4}, \mathbf{5}, \mathbf{7}$ and their image points are computed. For each noise level, we perform 100 runs, and the averaged results are calculated, still denoted by $I_{1}, I_{2}$. Since $\mathbf{1}, \mathbf{2}, \mathbf{3}, \mathbf{4}, \mathbf{5}, \mathbf{7}, \mathbf{O}$ lie on a twisted cubic and $\mathbf{1}, \mathbf{2}, \mathbf{3}, \mathbf{4}, \mathbf{5}, \mathbf{6}, \mathbf{O}$ do not lie on a twisted cubic, $I_{2}$ should be close to zero, while $I_{1}$ should not. And therefore there should be $I_{1}>I_{2}$.
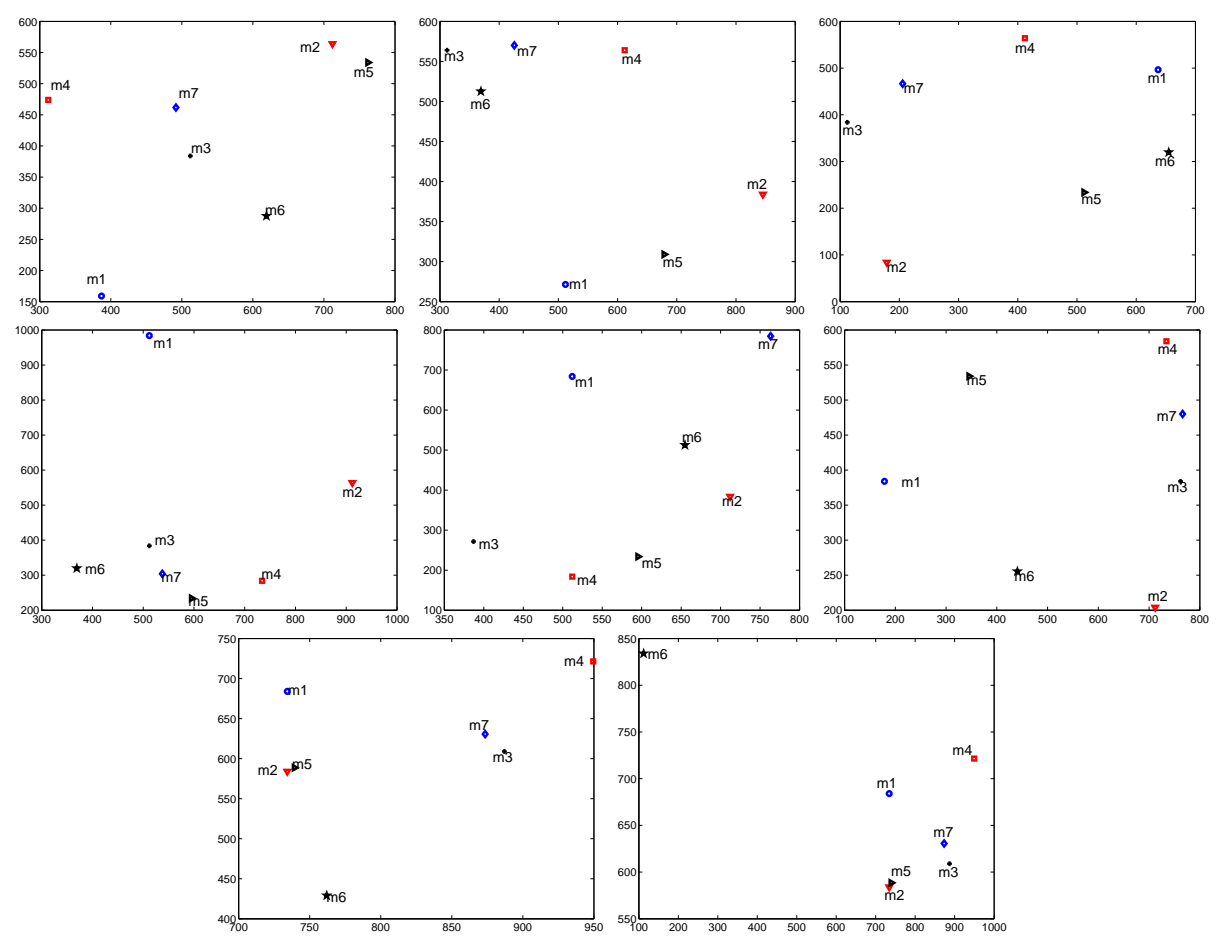

Fig. 1. Some image data, denoted as $D_{i}, i=1 . .8$, where $\mathbf{m}_{2}$ and $\mathbf{m}_{5}$ are very close in $D_{7}$ and $D_{8}$

We do the repeated simulations, the image sizes are not greater than $1000 \times$ 1000 pixels. We find that $I_{1}$ and $I_{2}$ are all very stable, and there are always $I_{1}>1.1>I_{2}$. Some image data $D_{i}, i=1 . .8$ are shown in Fig. 1 , where the images of $\mathbf{m}_{i}, i=1 . .5$ in $D_{i}, i=1 . .6$ distribute rather evenly, and the ones in $D_{7}, D_{8}$ do not $\left(\mathbf{m}_{2}, \mathbf{m}_{5}\right.$ are very close). The corresponding results of $I_{1}, I_{2}$ of them are shown in Table 1. 
Though there is noise, the variations of $I_{1}, I_{2}$ are very small, and there are always $I_{1}>I_{2}$. These show that the proposed algorithm can distinguish robustly between the twisted cubic degenerate configuration and the nondegenerate configuration, and the criterion function $f$ is quite stable against noise.

Table 1. The values $I_{1}, I_{2}$ under different noise levels

\begin{tabular}{ll|lllll}
\hline Noise level (pixel) & & 0 & 0.5 & 1 & 1.5 & 2 \\
\hline$D_{1}$ & $I_{1}$ & 2.41 & 2.41 & 2.41 & 2.41 & 2.41 \\
& $I_{2}$ & 0.00 & 0.00 & 0.00 & 0.00 & 0.00 \\
\hline$D_{2}$ & $I_{1}$ & 2.94 & 2.94 & 2.94 & 2.93 & 2.94 \\
& $I_{2}$ & 0.00 & 0.00 & 0.01 & 0.03 & 0.05 \\
\hline$D_{3}$ & $I_{1}$ & 2.67 & 2.67 & 2.67 & 2.67 & 2.67 \\
& $I_{2}$ & 0.00 & 0.00 & 0.00 & 0.00 & 0.00 \\
\hline$D_{4}$ & $I_{1}$ & 2.19 & 2.18 & 2.18 & 2.17 & 2.17 \\
& $I_{2}$ & 0.00 & 0.01 & 0.00 & 0.01 & 0.01 \\
\hline$D_{5}$ & $I_{1}$ & 2.86 & 2.86 & 2.86 & 2.86 & 2.86 \\
& $I_{2}$ & 0.00 & 0.00 & 0.00 & 0.00 & 0.00 \\
\hline$D_{6}$ & $I_{1}$ & 2.25 & 2.25 & 2.25 & 2.25 & 2.25 \\
& $I_{2}$ & 0.00 & 0.00 & 0.00 & 0.01 & 0.01 \\
\hline$D_{7}$ & $I_{1}$ & 2.75 & 2.77 & 2.76 & 2.77 & 2.75 \\
& $I_{2}$ & 0.00 & 0.11 & 0.25 & 0.48 & 0.52 \\
\hline$D_{8}$ & $I_{1}$ & 2.55 & 2.55 & 2.52 & 2.48 & 2.46 \\
& $I_{2}$ & 0.00 & 0.14 & 0.30 & 0.54 & 0.64 \\
\hline
\end{tabular}

\subsection{Experiments from Real Data}

This section will show the usefulness of the proposed criterion function $f$ that can be faithfully trusted for camera parameter estimation from six points in practice.

We are to calibrate a camera from a single view of a grid. We choose two groups of six pairs of space and image points with larger value and smaller value of the criterion function $f$, then from them, estimate camera parameters and compare the results.

The used image taken by a CCD camera is shown in Fig. 2. The size of the image is of $1024 \times 768$ pixels. We extract the pixels of the edges by Canny edge detector, then fit them as lines, and calculate the intersection points of these lines. The world coordinate system is set up in the grid. Then we have 108 pairs of space points and the corresponding image points. By using DLT method [1] from these 108 pairs of space and image points, we obtain the camera intrinsic 


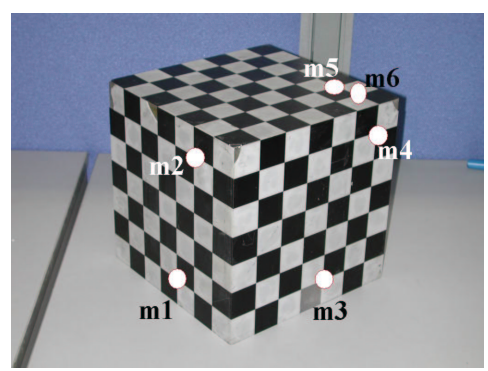

Fig. 2. A real image of a calibration grid, where $\mathbf{m}_{i}, \mathbf{i}=1 . .6$ are the image points from $G_{\min }$ and $G_{\min }$ is the group of six pairs of space and image points with the minimal value of the criterion function $f$

parameter matrix $\mathbf{K}$ and the camera pose parameters: rotation $\mathbf{R}$ and translation t as follows:

$\mathbf{K}=\left(\begin{array}{ccc}2049.8128 & -2.7983 & 523.9202 \\ 0 & 2050.5605 & 394.1385 \\ 0 & 0 & 1\end{array}\right), \mathbf{R}=\left(\begin{array}{ccc}0.7784 & -0.6272 & 0.0270 \\ -0.2648 & -0.3671 & -0.8917 \\ 0.5692 & 0.6870 & -0.4518\end{array}\right)$, $\mathbf{t}=(-0.7503,4.8624,30.7296)^{T}$.

From these 108 pairs of space and image points, we randomly combine 125 groups of six pairs with no three image points collinear, no three space points collinear, and no four space points coplanar. We choose the group with the maximal value of the criterion function $f$, the group with the minimal value of the criterion function $f$, and denote them as $G_{\max }, G_{\min }$ respectively. The value of $f$ from $G_{\max }$ is 3.0997, and the value of $f$ from $G_{\min }$ is 0.0380 .

The image points of $G_{\min }$ are plotted as $\mathbf{m}_{i}, i=1 . .6$ as shown in Fig. 2 . We can see that there is no linear relation among them. The value of $f, 0.0380$, says that they are from degenerate configuration.

We calibrate the camera from the six pairs of space and image points in $G_{\max }$ by DLT method, and the results are: $\mathbf{K}_{1}=\left(\begin{array}{ccc}2140.9987 & -2.1069 & 570.3262 \\ 0 & 2138.6413 & 452.6338 \\ 0 & 0 & 1\end{array}\right), \mathbf{R}_{1}=\left(\begin{array}{ccc}0.7668 & -0.6409 & 0.0358 \\ -0.2772 & -0.3808 & -0.8822 \\ 0.5790 & 0.6665 & -0.4696\end{array}\right)$,
$\mathbf{t}_{1}=(-1.4424,3.9693,32.1664)^{T}$.

Similarly, we calibrate the camera from the six pairs of space and image points in $G_{\min }$, and the results are:

$$
\begin{aligned}
& \mathbf{K}_{2}=\left(\begin{array}{ccc}
980.4078 & 26.8782 & 430.9372 \\
0 & 870.5114 & 541.8497 \\
0 & 0 & 1
\end{array}\right), \mathbf{R}_{2}=\left(\begin{array}{ccc}
-0.6666 & 0.7454 & 0.0062 \\
-0.0205 & -0.0266 & 0.9994 \\
0.7451 & 0.6661 & 0.0330
\end{array}\right), \\
& \mathbf{t}_{2}=(-1.0456,-1.5375,-18.0317)^{T} .
\end{aligned}
$$

We evaluate the estimated $\mathbf{K}_{1}$ and $\mathbf{K}_{2}$ by comparing them with $\mathbf{K}$ :

$\mathbf{K}_{1}-\mathbf{K}=\left(\begin{array}{ccc}91.1859 & 0.6914 & 46.4060 \\ 0 & 88.0809 & 58.4953 \\ 0 & 0 & 0\end{array}\right)$ 
$\mathbf{K}_{2}-\mathbf{K}=\left(\begin{array}{ccc}-1069.4050 & 29.6765 & -92.9830 \\ 0 & -1180.0491 & 147.7112 \\ 0 & 0 & 0\end{array}\right)$

It is clear that the absolute error for each intrinsic parameter from $\mathbf{K}_{2}$ is much greater than each one from $\mathbf{K}_{1}$. We evaluate the recovered $\mathbf{R}_{1}$ and $\mathbf{R}_{2}$ by comparing them with $\mathbf{R}$ :

$\mathbf{R}_{1}-\mathbf{R}=\left(\begin{array}{ccc}-0.0116 & -0.0137 & 0.0087 \\ -0.0123 & -0.0137 & 0.0095 \\ 0.0098 & -0.0205 & -0.0178\end{array}\right), \mathbf{R}_{2}-\mathbf{R}=\left(\begin{array}{ccc}-1.4450 & 1.3725 & -0.0208 \\ 0.2443 & 0.3405 & 1.8911 \\ 0.1759 & -0.0208 & 0.4848\end{array}\right)$ And also, the recovered $\mathbf{t}_{1}$ and $\mathbf{t}_{2}$ are evaluated by the differences between them and $\mathbf{t}$ :

$\mathbf{t}_{1}-\mathbf{t}=(-0.6922,-0.8931,1.4368)^{T}, \mathbf{t}_{2}-\mathbf{t}=(-0.2954,-6.3999,-48.7613)^{T}$. Also, it is clear that the accuracies of $\mathbf{R}_{1}, \mathbf{t}_{1}$ are higher than the accuracies of $\mathbf{R}_{2}, \mathbf{t}_{2}$ except the first element of the translation. For the first element of the translation, the absolute error from $\mathbf{t}_{1}$ is greater than the absolute error from $\mathbf{t}_{2}$, but the difference between these two absolute errors is not so large as that for the second or third element of the translation.

So, we can see that the calibration result from six pairs of space and image points with smaller value of the criterion function $f$ (i.e. space points and optical center are near to the twisted cubic degenerate configuration) is not better than the one from six pairs of space and image points with larger value of the criterion function $f$ (i.e. space points and optical center are far from the twisted cubic degenerate configuration). The proposed criterion function $f$, thus, can be faithfully trusted for camera parameter estimation from six points. We also perform the experiments from other real images and obtain the similar results. The details are omitted due to the space limit.

\section{Summary and Conclusions}

We establish the invariant relationship between six space points and their images under a perspective view when camera optical center and the space points lie on a twisted cubic. Then, the invariant relationship is used to recognize the nontrivial degenerate configuration of six points through a new algorithm. The algorithm is based on a criterion function, does not need explicit computations on the optical center or projective matrix, and is shown stable and robust against noise. We believe that it has further usefulness. For example, when applying RANSAC during the process of determining camera parameters, the critical groups of data can be filtered by the criterion function of this method, and then the algorithm can be extended to more than six pairs of space points and image points. The sample of six pairs of space points and image points with poor performance will not be chosen in RANSAC. How to know whether a sample is unreliable or not? The criterion function in this paper just can be used to detect the unreliability. We will report this work in future. The invariant relationship can also be easily extended to the the invariant relationship between two perspective views when the two camera optical centers and space points lie on the same twisted cubic, 
and then the result can similarly be used to detect critical data for computing fundamental matrix or epipoles $[6,8]$.

\section{Acknowledgments}

This work was supported by the National Natural Science Foundation of China under grant No. 60303021 and No. 60475009.

\section{References}

1. Abdel-Aziz, Y.I., Karara, H.M.: Direct Linear Transformation from Comparator Coordinates into Object Space Coordinates in Close-range Photogrammetry. Proc. ASP/UI Symp. on Close Range Photogrammetry (1971)1-18.

2. Bayro-Corrochano, E., Banarer, V.: A Geometric Approach for the Theory and Applications of 3D Projective Invariants. Journal of Mathematical Imaging and Vision, Vol. 16, No. 2 (2001)131-154.

3. Carlsson, S.: Symmetry in Perspective. ECCV (1998)249-263.

4. Carlsson, S.: View Variation and Linear Invariants in 2-D and 3-D. Tech. Rep. ISRN KTH/NA/P-95/22-SE, Dec. (1995).

5. Carlsson, S.: The Double Algebra: An Effective Tool for Computing Invariants in Computer Vision. Applications of Invariance in Computer Vision: Joint EuropeanUS Workshop, Azores, Portugal, Oct. (1993)145-164.

6. Hartley, R., Zisserman, A.: Multiple View Geometry in Computer Vision. Cambridge University Press (2000).

7. Hartley, R.: Projective Reconstruction and Invariants from Multiple Images. IEEE Trans. Pattern Analysis and Machine Intelligence, Vol. 16, No. 10 (1994)1036-1041.

8. Maybank, S.: Theory of Reconstruction from Image Motion. Springer-Verlag (1993).

9. Maybank, S.: Relation between 3D Invariants and 2D Invariants. IEEE Workshop on Representation of Visual Scenes, Cambridge, Massachusetts, Jun. (1995).

10. Quan, L.: Invariants of 6 Points from 3 Uncalibrated Images. ECCV (1994)459-470.

11. Quan, L.: Invariants of Six Points and Projective Reconstruction from Three Uncalibrated Images. IEEE Trans. Pattern Analysis and Machine Intelligence, Vol. 17, No. 1 (1995)34-46.

12. Roh, K.S., Kweon, I.S.: 3-D Object Recognition Using a New Invariant Relationship by Single-view. Pattern Recognition, Vol. 33, No. 5 (2000)741-754.

13. Schaffalitzky, F., Zisserman, A., Hartley, R., Torr, P.: A Six Point Solution for Structure and Motion. ECCV (2000)632-648.

14. Semple, J., Kneebone, G.: Algebraic Projective Geometry. Oxford University Press (1952).

15. Weng, J., Huang, T.S., Ahuja, N.: Motion and Structure from Two Perspective Views: Algorithms, Error Analysis, and Error Estimation. IEEE Trans. Pattern Analysis and Machine Intelligence, Vol. 11, No. 5 (1989)451-476.

16. Wu, Y.H., Hu, Z.Y.: The Invariant Representations of a Quadric Cone and a Twisted Cubic. IEEE Trans. Pattern Analysis and Machine Intelligence, Vol. 25, No. 10 (2003)1329-1332.

17. Wu, Y.H., Hu, Z.Y.: A Unified and Complete Framework of Invariance for Six Points. In: Li, H., Olver, P.J., and Sommer, G. (Eds.), Computer Algebra and Geometric Algebra with Applications, IWMM/GIAE 2004, Springer-Verlag, LNCS 3519 (2005)403-417. 